University of Nebraska - Lincoln

DigitalCommons@University of Nebraska - Lincoln

9-1998

\title{
Optimizing Amplifier Placements in a Multiwavelength Optical LAN/MAN: The Equally Powered-Wavelengths Case
}

Byrav Ramamurthy

University of Nebraska-Lincoln, bramamurthy2@unl.edu

Jason Iness

IEEE

Biswanath Mukherjee

IEEE

Follow this and additional works at: https://digitalcommons.unl.edu/csearticles

Part of the Computer Sciences Commons

Ramamurthy, Byrav; Iness, Jason; and Mukherjee, Biswanath, "Optimizing Amplifier Placements in a Multiwavelength Optical LAN/MAN: The Equally Powered-Wavelengths Case" (1998). CSE Journal Articles. 71.

https://digitalcommons.unl.edu/csearticles/71

This Article is brought to you for free and open access by the Computer Science and Engineering, Department of at DigitalCommons@University of Nebraska - Lincoln. It has been accepted for inclusion in CSE Journal Articles by an authorized administrator of DigitalCommons@University of Nebraska - Lincoln. 


\title{
Optimizing Amplifier Placements in a Multiwavelength Optical LAN/MAN: The Equally Powered-Wavelengths Case
}

\author{
Byrav Ramamurthy, Jason Iness, and Biswanath Mukherjee, Member, IEEE
}

\begin{abstract}
Wavelength division multiplexing (WDM) provides the ability to utilize the enormous bandwidth offered by optical networks, using today's electronics. WDM-based optical networks employing passive-star couplers have been proposed for deployment in local and metropolitan areas. Optical amplification is often required in such networks to compensate for the signal attenuation along the fiber links and the splitting and coupling losses in the network. However, an optical amplifier has constraints on the maximum gain and the maximum output power it can supply; thus optical amplifier placement becomes a challenging problem. A simplifying assumption for analytical tractability requires that all wavelengths, present at a particular point in a fiber, be at the same power level, viz. the equally powered-wavelengths case. However, previous studies did not minimize the total number of amplifiers while achieving power equalization. In this paper, we formulate the minimization of amplifiers with power equalization as a mixed integer linear program (MILP) that can be solved by a linear program solver. Illustrative examples on sample networks are presented, which demonstrate the characteristics and the advantages of our optimal amplifier placement algorithm.
\end{abstract}

Index Terms - Amplifier placement, LAN/MAN, linear programming, optical network, optimization, passive star, power equalization, WDM.

\section{INTRODUCTION}

$\mathbf{S}$ EVERAL architectures have been proposed for broadband optical local/metropolitan area networks and access networks [1]-[6]. Wavelength division multiplexing (WDM) is used in such networks to support high-bandwidth applications. In this study, we focus on a class of such networks, which enables stations to broadcast along a dedicated wavelength (lightpath) to all other stations in the network. We describe below the structure of this network in detail. Consider an optical local/metropolitan area network (LAN/MAN) (also referred to as optical access networks, passive optical networks (PON's), etc.) as shown in Fig. 1. The network consists of $N$

Manuscript received May 22, 1998. This work was supported in part by the National Science Foundation (NSF) under Grant NCR-92-05755 and ECS95-21249 and in part by Advanced Research Projects Agency (ARPA) under Contract DABT63-92-C-0031.

B. Ramamurthy is with the University of Nebraska at Lincoln, Lincoln, NE 68508 USA (e-mail: byrav@cse.unl.edu).

J. Iness was with the Department of Computer Science, University of California, Davis, CA 95616 USA. He is now with Intel Corporation, Portland, OR USA.

B. Mukherjee is with the Department of Computer Science, University of California, Davis, CA 95616 USA (e-mail: mukherjee@cs.ucdavis.edu).

Publisher Item Identifier S 0733-8724(98)06683-3. stations and $M$ passive optical star couplers ("stars"), which are of the broadcast and "nonreflective" type (see below) in order to prevent loops in the network. Further, the stars, and the stations together form a "tree" (which is a "loopless" network).

Each station has a fixed-wavelength transmitter which operates on its own unique wavelength channel. Each station either has a tunable receiver or a receiver array in order to receive signals from all of the other stations. A station's transmission can be received by every other station after being subject to losses and gains as the signal traverses through different parts of the network. Each "nonreflective star" consists of pairs of inputs and outputs, and each output carries all of the wavelengths that were incident on all of the inputs except for the wavelengths that were carried on its own paired input, as in the Level-0 all-optical network (AON) [7]. In a $k \times k$ star, the power on each wavelength on an input fiber is directed to the other $k-1$ output fibers and divided evenly among them. This is referred to as the splitting loss at a star. Each transmitted signal/wavelength must be received at all of the other receivers at a power level greater than a station's receiver sensitivity level, denoted by $p_{\text {sen }}$. Apart from the splitting loss at a star, there is signal attenuation on the fibers given by the parameter $\alpha \mathrm{dB} / \mathrm{km}$. Although fiber has low attenuation, large networks (MAN's) and networks with numerous splitting/coupling losses may require amplification to allow a signal to reach the receivers at a detectable level. Since optical amplifiers are costly, we should minimize their number in the network. Reducing the number of amplifiers is also important based on noise, maintenance, and faulttolerance considerations.

The constraints on the system are shown in Table I, along with typical values for each parameter. $P_{\text {NONLIN,max }}$ defines the power level, in a fiber, above which a signal encounters significant nonlinear effects. However, the total power at any point in the network is usually bounded by a lower value $P_{\max }$, which is the maximum output power of an amplifier and a transmitter. $P_{\text {sat }}$ is the internal saturation power of the optical amplifier. $G_{\max }$ is the maximum small-signal gain of the optical amplifier. These parameter values (last column of Table I) will be used in our illustrative numerical examples in Section III.

The value of the parameter $p_{\text {sen }}$ can be chosen (by the user) such that it remains much higher than the noise levels at the intermediate amplifiers and at the receiver. The value of $p_{\text {sen }}$ can also be changed iteratively, after the placement 


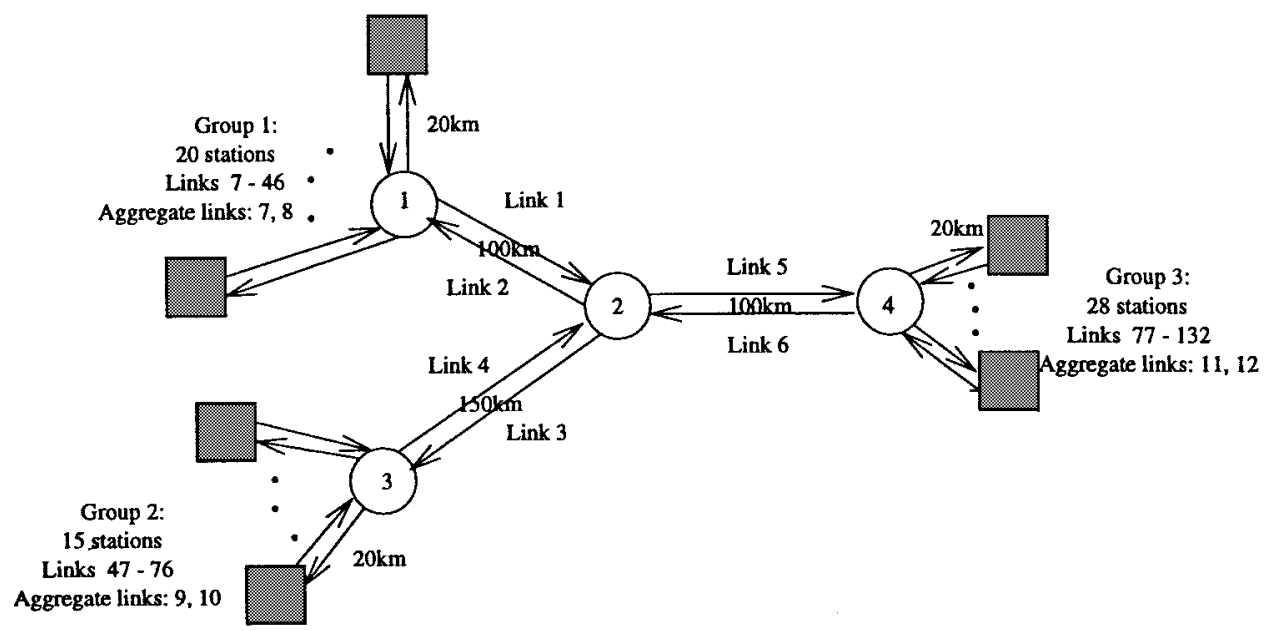

Fig. 1. Example of a passive-star-based optical metropolitan area network (slightly modified version of the one used in [3]).

TABLE I

Important Parameters and Their Values Used in the Amplifier-Placement Algorithms

\begin{tabular}{l|l|l|l}
\hline Parameter & Description & Range & Value used \\
\hline$p_{\text {sen }}$ & $\begin{array}{l}\text { Minimum signal power at receiver } \\
\text { and the amplifier sensitivity level }\end{array}$ & $-30 \mathrm{dBm}$ at $1 \mathrm{Gbps}$ & $-30 \mathrm{dBm}$ \\
\hline$G_{\max }$ & Maximum small-signal gain & $\leq 25 \mathrm{~dB}(\mathrm{MQW})[8]$ & $20 \mathrm{~dB}$ \\
\hline$P_{N O N L I N, \max }$ & Maximum total power in fiber & $10-17 \mathrm{dBm}$ & $10 \mathrm{dBm}$ \\
\hline$P_{m a x}$ & $\begin{array}{l}\text { Maximum total output power of amplifier } \\
\text { and transmitter }\end{array}$ & & $0 \mathrm{dBm}$ \\
\hline$P_{\text {sat }}$ & Internal saturation power of the amplifier & & \\
\hline$\alpha$ & Fiber attenuation & & $1.55 \mathrm{dBm}$ \\
\hline
\end{tabular}

of the amplifiers, in order to obtain the desired bit error rate (BER) at each of the receivers, following the approach in [3]. Thus, in this study, we do not consider system factors such as amplifier ASE noise and crosstalk at the receivers, explicitly; these factors are assumed to be implicitly incorporated in the parameter $p_{\text {sen }}$.

As noted in [9], standardization efforts are being undertaken, by international bodies such as ITU-T, for point-to-point WDM line systems and for optical networks. Parameters such as optical fiber span lengths between amplifiers, recommended amplifier gains and optical channel frequencies are expected to be specified in these standards. These parameter values may be incorporated in the design of long-haul transmission systems readily to benefit from the availability of standardized equipment. However, for custom-built networks, such as those discussed in this paper, there is a lot of savings to be obtained by computing the optimal number of amplifiers needed to operate the network, without additional restrictions on amplifier spacing, gain, etc. Note that the results from the approach outlined in this paper can serve as a good lower bound for any amplifier-placement algorithm, which includes these additional restrictions, for such networks.

\section{A. Problem Definition}

Our goal is to find the minimum number of amplifiers required to operate the network and to determine their exact placements. In general, when signals on different wavelengths originating from different transmitters arrive at an amplifier, their power levels could be very different. This phenomenon is known as the near-far effect and it may result in inefficient utilization of an individual amplifier. Due to the difference in power levels, the higher-powered wavelengths could saturate the amplifier and limit the gain seen by the lower-powered wavelengths. Moreover, allowing wavelengths in the same fiber to be at different power levels results in a mixed-integer nonlinear program [10] for the optimal amplifier placement problem, and it cannot be solved exactly.

These problems can be bypassed by restricting all of the wavelengths at any given point in a fiber to be at the same power level. In previous work [3], the amplifier-placement scheme operated on a link-by-link basis by maintaining the power level of all the wavelengths at the output of each star at the lowest possible value (i.e., the receiver sensitivity level, $\left.p_{\text {sen }}\right)$. An improved method would be to formulate and solve the amplifier-placement problem as a global optimization 


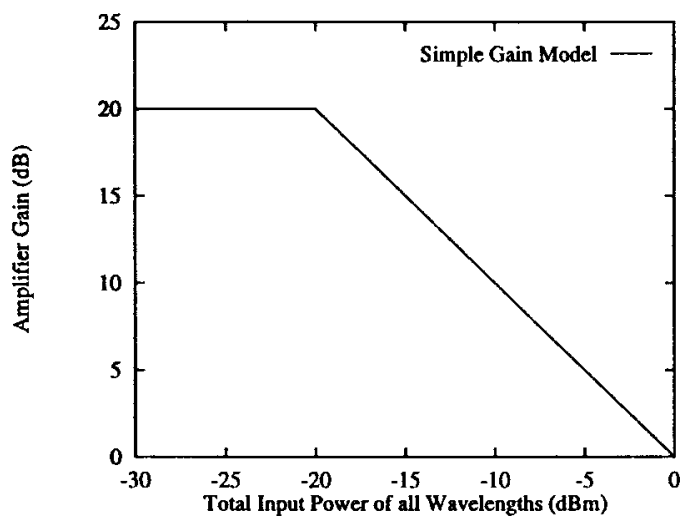

Fig. 2. Original amplifier gain model approximations used in previous studies [3].

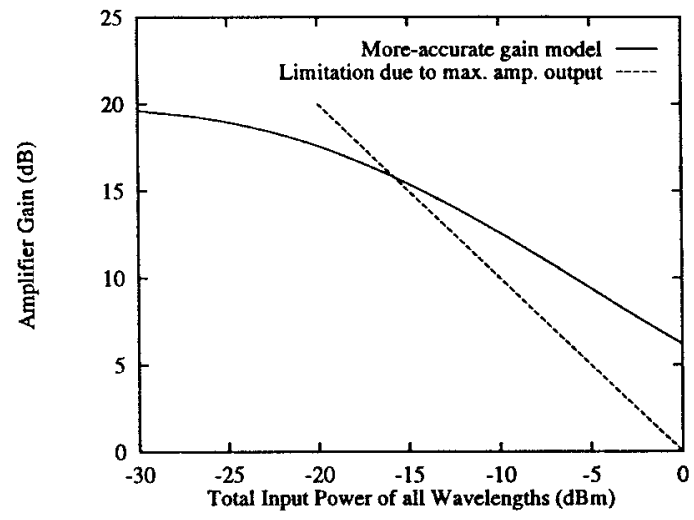

Fig. 3. More-accurate amplifier gain model used in this study.

problem, while retaining the constraint on equal powers on all wavelengths.

Our paper presents such a method to minimize the number of optical amplifiers. The method works as follows: 1) determine whether or not it is possible to design the network taking into consideration the limitations of the devices (e.g., the power budget of the amplifiers), 2) generate a linear set of constraints to describe the problem setting, 3) use a mixedinteger linear program (MILP) solver to determine the global minimum number of amplifiers needed across the network, and 4) determine the exact placements of the amplifiers. Numerical examples will show that this global optimization method can result in a significant savings in the number of amplifiers over the link-by-link approach in [3].

\section{B. Amplifier Gain Model}

Previous studies [3] used the gain model in Fig. 2. In this model, it is assumed that the full small-signal gain is realizable until the point at which the amplifier output becomes power-limited. At this point, the amplifier is assumed to enter saturation and the gain starts to drop. This "point" of saturation occurs in the example of Fig. 2 at a total input power of -20 $\mathrm{dBm}$. At lower input powers, the amplifier is assumed to be able to supply the full small-signal gain of $G_{\max }=20 \mathrm{~dB}$.
A more-accurate amplifier model is given by (from [11])

$$
\frac{P_{\text {in }}}{P_{\text {sat }}}=\frac{1}{G-1} \ln \left(\frac{G_{0}}{G}\right)
$$

where $P_{\text {in }}$ is the total input power (across all wavelengths) to the amplifier, $P_{\text {sat }}$ is the internal saturation power, $G$ is the actual gain achieved, and $G_{0}$ is the small-signal gain. (Note that all values are in absolute scale, not $\mathrm{dB}$.) This model, which is plotted as a solid line in Fig. 3, shows how saturation does not happen at a specific point but is really a continuous effect. In fact, even for small input powers, the amplifier is not able to supply the full small-signal gain of $G_{\max }=20 \mathrm{~dB}$. At high input powers, the gain available at the amplifier is limited to the saturation output power, $P_{\max }$, as shown by the dashed lines in Fig. 3. The value of $P_{\max }$ is retained as $1 \mathrm{~mW}(0$ $\mathrm{dBm})$ as in earlier work [3], in order to facilitate comparison of the two amplifier-placement algorithms. Hence, the model used throughout this paper, follows the solid line in Fig. 3 for low-input powers and the dashed line for high-input powers.

However, our gain model of a generic optical amplifier presented here does include some simplifying assumptions. We assume that all wavelengths contribute equally to the gain saturation of the amplifier. Hence, we can aggregate the input powers on the individual wavelengths to determine the available gain from Fig. 3. While this assumption is acceptable for wavelengths which are close together in the spectrum, it is an approximation for wavelengths which are wide apart [12]. Handling wavelength-dependent gain, gain saturation, and noise characteristics as described in [13] remains an area of future work.

\section{SOLUTION APPROACH}

Our solution approach consists of four modules. The interaction between the various modules is shown in Fig. 4.

- Module I. Test the feasibility of the network.

- Module II. Generate the constraints.

- Module III. Solve the mixed-integer linear program (MILP)

- Module IV. Place the amplifiers.

The functionality of the modules is described in Sections IIB-II-E, after describing the notation in Section II-A. Section II-F derives a lower bound on the solution (minimum number of amplifiers required) to be $M-1$, where $M$ is the number of stars in the network.

\section{A. Notation}

The variables in boldface are dependent on the specific amplifier-placement algorithm used. The other variables are fixed for a given network topology. Unless otherwise stated, all calculations are performed in the $d B$ scale; hence, the absolute power levels are measured in $\mathrm{dBm}$ and the gains in $\mathrm{dB}$.

\section{1) Device Parameters:}

- $p_{\text {sen }}=$ minimum power required on a wavelength for detection in $\mathrm{dBm}$. This represents both the receiver sensitivity level and the amplifier sensitivity level, which have been assumed to be equal. 


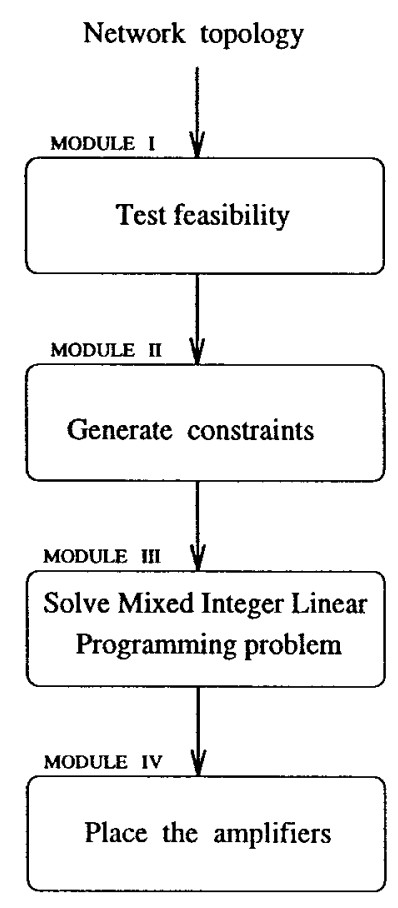

Fig. 4. Modules.

- $P_{\max }=$ maximum power available from an amplifier in $\mathrm{dBm}$

$=$ maximum power of a transmitter in $\mathrm{dBm}$.

It is not necessary that the maximum available power at an amplifier and the maximum transmitter power be identical. For simplicity, we have assumed them to be equal.

- $G_{\max }=$ maximum (small-signal) amplifier gain in $\mathrm{dB}$.

- $\alpha=$ signal attenuation in $\mathrm{dB} / \mathrm{km}$.

2) Network-Wide Variables:

- $N=$ number of access stations in the network

$=$ number of wavelengths in the network.

- $M=$ number of stars in the network.

- $L=$ number of links in the network (including both access links and links between stars).

- $\mathbf{A}=$ number of amplifiers in the network.

3) Link Variables: The variables associated with link $l, 1 \leq$ $l \leq L$ are the following.

- $L_{l}=$ length of link $l$ in $\mathrm{km}$.

- $\lambda_{l}=$ set of wavelengths carried by link $l$.

Hence, $\left|\lambda_{l}\right|=$ number of wavelengths carried by link $l$.

- $\operatorname{gmax}_{l}=$ maximum per-wavelength gain that can be offered by an amplifier on link $l$. Note that, if a link carries many wavelengths, its $\operatorname{gmax}_{l}$ may be less than the smallsignal gain, $G_{\max }$. This is due to the fact that an amplifier has a limited maximum output power (aggregated over all wavelengths) which in turn may limit $\operatorname{gmax}_{l}$.

- $\mathrm{n}_{\mathbf{l}}=$ number of amplifiers on link $l$.

- $\mathbf{S G}_{\mathbf{l}}=$ total gain required on link $l$ as determined by the amplifier-placement algorithm.

4) Star Variables: The variables associated with star $i, 1 \leq$ $i \leq M$, are the following.

- $D_{i}=$ degree of star $i$.
Hence, the splitting loss in $\mathrm{dB}$, as seen by a signal on an input link to star $i=10 \log _{10}\left(D_{i}-1\right)$, because of the nonreflective nature of the stars, as mentioned earlier.

- $\mathbf{p}_{\mathbf{i}}=$ output power of star $i$ on each wavelength.

\section{B. Module I: Test the Feasibility of the Network}

We first determine whether the given network has a feasible amplifier placement. The feasibility test requires that all of the signals from all of the transmitters should be able to reach all of the destinations at a sufficiently high power level for detection. Furthermore, this should be carried out without exceeding the capacities of the transmitters and the amplifiers. The attenuation loss suffered by the signals on any link can be compensated for by using an adequate number of optical amplifiers. However, in some cases, we may not be able to compensate for the huge splitting loss at a star with a large fanout, even by placing an amplifier at each input to the star. This is due to the fact that we employ only "passive" optical stars in the network, i.e., there is no scope for signal amplification within a star.

As shown in [3], we only need to ensure that the power level of each input signal on every input link to a star is high enough to guarantee that the signal exits the star on every output link at a power level of at least $p_{\text {sen }}$. However, the power level at the input cannot exceed the maximum possible power supplied by an amplifier over all wavelengths, which we limit to $P_{\max }$. Hence, we subtract the star's splitting loss from the maximum possible per-wavelength power at the input to obtain the corresponding per-wavelength output power and compare it against $p_{\text {sen }}$. Given that link $j$ contains $\left|\lambda_{j}\right|$ wavelengths, and that the power on each of these channels must be split $\left(D_{i}-1\right.$ ) ways at star $i$ (of degree $D_{i}$ ), the test for feasibility can thus be written as follows:

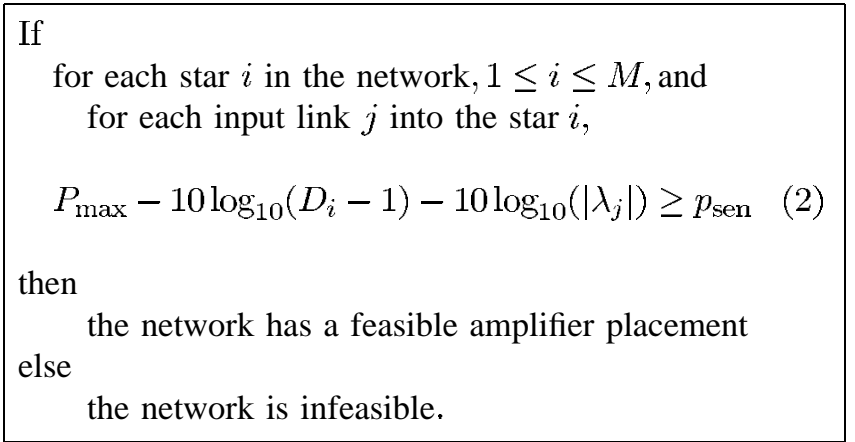

We can reduce the computation in the above test by identifying the star $i_{\max }$ and its adjacent input link $j_{\max }$ such that the product $\left(D_{i_{\max }}-1\right)\left(\left|\lambda_{j_{\max }}\right|\right)$ is the maximum among all such pairs in the network. Now, this star-link pair has the greatest amount of loss that needs to be compensated for by an amplifier. Hence, the network is feasible if (2) holds for $i=i_{\max }$ and $j=j_{\max }$, and is infeasible otherwise.

\section{Module II: Generate the Constraints}

This module generates the linear constraints on the amplifier placement for the given network topology. Since the number of wavelengths carried by a link is known a priori, the maximum 
gain offered by any amplifier on that link can be computed beforehand, as described below in Section II-C1, for each link in the network. We then use the values of $\operatorname{gmax}_{l}$ to specify the problem's constraints in Sections II-C2-II-C5. We remark that all power levels except $P_{\max }$ are given on a per-wavelength basis.

1) Precomputation of gmax: The maximum gain available at an amplifier $\left(g_{\max }\right)$ along any link $l$ can be computed off-line when all the powered wavelengths at any particular point in the network carry equal power. From the models in Figs. 2 and 3, it is clear that the available gain at an amplifier is a function of the total input power aggregated across all wavelengths, the maximum small-signal gain of the amplifier $\left(G_{\max }\right)$, and the total internal saturation power of the amplifier $\left(P_{\text {sat }}\right)$. Further, this gain is maximum when the input signals are at the lowest possible levels, viz. $p_{\text {sen }}$. Now, if $P_{l}^{\min }$ denotes the total power, in $\mathrm{dB}$, across all wavelengths when all the input signals are at $p_{\text {sen }}$, we have, for any link $l$

$$
\max _{l}=G\left(P_{l}^{\min }, G_{\max }, P_{\mathrm{sat}}\right) \text {. }
$$

We remark that various amplifier gain models can be used to obtain this function $G$.

For any link $l$ which connects star $i$ to star $j$, as shown in Fig. 5, there are $\left|\lambda_{l}\right|$ wavelengths in the link and hence the minimum total power across all these wavelengths is given by

$$
P_{l}^{\min }=p_{\text {sen }}+10 \log _{10}\left(\left|\lambda_{l}\right|\right)
$$

and hence we have [from (3)]

$$
\operatorname{gmax}_{l}=G\left(p_{\mathrm{sen}}+10 \log _{10}\left(\left|\lambda_{l}\right|\right), G_{\max }, P_{\text {sat }}\right) .
$$

Similarly, for any link $l$ which connects station $k$ to star $j$ (and hence carries only one wavelength, viz. the one originating at station $k$ ), as shown in Fig. 6, we have

$$
g \max _{l}=G\left(p_{\mathrm{sen}}, G_{\max }, P_{\mathrm{sat}}\right) \text {. }
$$

Finally, for any link $l$, from star $i$ to station $k$ (which carries all wavelengths destined to station $k$, except its own), as shown in Fig. 7, we have

$$
g \max _{l}=G\left(p_{\text {sen }}+10 \log _{10}(N-1), G_{\max }, P_{\text {sat }}\right) .
$$

2) Link Constraints: For any link $l$ from star $i$ to star $j$ as shown in Fig. 5, we can bound the power $p_{j}$ at any of star $j$ 's output by star $i$ 's output power $p_{i}$ and the gain available on link $l$, as follows:

$$
p_{i}-\alpha \cdot L_{l}-10 \log _{10}\left(D_{j}-1\right)+S G_{l}=p_{j}
$$

where the two loss terms correspond to attenuation loss (in $L_{l}$ $\mathrm{km}$ of fiber) and a $\left(D_{j}-1\right)$ way splitting loss at star $j$ while $S G_{l}$ is the total gain supplied by the $n_{l}$ amplifiers on link $l$. Since we want to provide the gain using as few amplifiers as necessary, we require that

$$
S G_{l} \leq g \max x_{l} \cdot n_{l}
$$

and

$$
S G_{l}>g \max l \cdot\left(n_{l}-1\right) .
$$

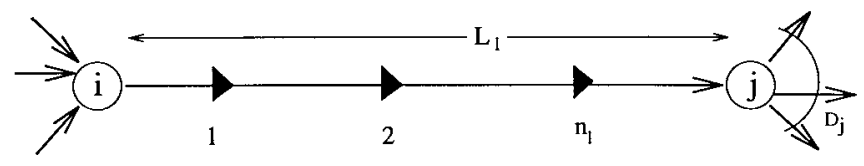

Fig. 5. Link between stars.

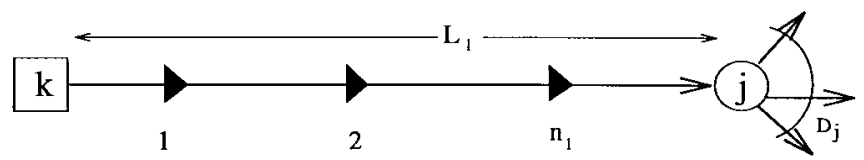

Fig. 6. Link from a station to a star.

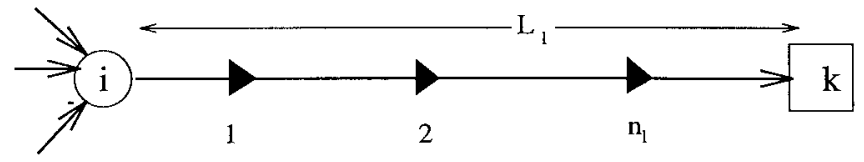

Fig. 7. Link from a star to a station.

Similarly, for any link $l$ from station $k$ to star $j$ as shown in Fig. 6, we can bound the power $p_{j}$ at any of star $j$ 's output by $P_{\max }$, the total power available at a transmitter, and by $\operatorname{gmax}_{l}$, the gain available on link $l$, as follows:

$$
P_{\max }-\alpha \cdot L_{l}-10 \log _{10}\left(D_{j}-1\right)+\operatorname{gmax}_{l} \cdot n_{l} \geq p_{j} \text {. }
$$

Finally, for any link $l$ from star $i$ to station $k$ as shown in Fig. 7, we require that the received power at a station on each wavelength be at least $p_{\text {sen }}$, the sensitivity level, i.e.

$$
p_{i}-\alpha \cdot L_{l}+\operatorname{gmax}_{l} \cdot n_{l} \geq p_{\text {sen }} .
$$

3) Star Constraints: For any star $i, 1 \leq i \leq M$, we require that the power at the output of the star on each wavelength be at least equal to $p_{\text {sen }}$, i.e.,

$$
p_{i} \geq p_{\text {sen }}
$$

The following constraints [(14) and (15)] are needed to completely specify the problem. For each star $j$, and for each incoming link $l$ into star $j$, we ensure that the required output power $p_{j}$ is feasible, i.e.

$$
P_{\max }-10 \log _{10}\left(D_{j}-1\right)-10 \log _{10}\left(\left|\lambda_{l}\right|\right) \geq p_{j} .
$$

Given a network configuration, the link that feeds in the maximum number of wavelengths to star $j$ is known a priori; so, if this link satisfies the above criterion, all other input links to star $j$ will satisfy it as well. Hence, for each star $j$, the above set of constraints in (14) reduces to the following single one:

$$
P_{\max }-10 \log _{10}\left(D_{j}-1\right)-10 \log _{10}\left(\left|\lambda_{l \max _{j}}\right|\right) \geq p_{j}
$$

where $l \max _{j}=$ index of the incoming link carrying the maximum number of wavelengths to star $j$.

4) Integrality Constraints: For each link $l$

$$
n_{l} \text { is an integer. }
$$

\section{5) Objective Function:}

$$
\text { Minimize } A=\sum_{l} n_{l} \text {. }
$$




\section{Module III: Solve the Mixed-Integer Linear Program (MILP)}

Note that the only variables in the above problem formulation are the $p_{i}$ 's, the per-wavelength output power at star $i$, the $n_{l}$ 's, the number of amplifiers on link $l$, and the $S G_{l}$ 's, the total gain required on link $l$. Thus, given that there are $M$ stars and $L$ links, the number of variables used is $O(M+L)$.

The constraints [(8)-(16)] are found to be linear, i.e, they can be written as a matrix inequality of the form $A x \leq B$. Moreover, the objective function is a linear function of the variables. However, because of the integrality constraint on the values of the $n_{l}$ 's [(16)], the problem is no longer a pure linear program (which it would have been if all of its variables, viz. $n_{l}$ 's, $p_{i}$ 's and $S G_{l}$ 's were real-valued); instead the integer $n_{l}$ 's make it a mixed-integer linear program (MILP).

The constraints generated by Module II can be fed to a MILP solver (e.g., lp_solve [14]) to obtain the optimum values of the $p_{i}$ 's and the $n_{l}$ 's. These MILP solvers typically employ branch-and-bound strategies [15] to handle the integrality constraints, thereby significantly cutting down the exponential search space.

\section{E. Module IV: Place the Amplifiers}

From the values of $p_{i}$ and $n_{l}$ obtained from Module III, we have for all the internal ("star-to-star") links in the network the following quantities:

- total and per-wavelength power $\left(p_{s r c}\right)$ of the signals at the source of the link;

- total and per-wavelength power $\left(p_{\text {dest }}\right)$ of the signals at the destination of the link;

- number of amplifiers $\left(n_{l}\right)$ needed on the link;

- total gain required on the link, $S G_{l}$.

However, on the access links from a station to the adjacent star, there is some flexibility in the transmitter power [see (11)]. Similarly, on the access links from a star to the adjacent stations, there is some flexibility in the received power [see (12)]. We operate the transmitters and receivers such that their power levels are kept at a minimum.

Now, we can place the amplifiers on link $l$ such that the first $\left(n_{l}-1\right)$ amplifiers in the downstream direction operate at a gain, $g \max _{l}$. The last amplifier operates at a gain, $S G_{l}-\left(n_{l}-1\right) \cdot \operatorname{gmax}_{l}$. Thus, the gain of each of the amplifier on the link is fixed; the only other parameters that needs to be specified are the exact locations of each amplifier. (Of course, other gain combinations of the amplifiers are also possible.)

We have some choices in determining where exactly the amplifiers are to be placed on the link. Below, we examine two different methods: ASAP and ALAP.

1) ASAP (As Soon As Possible): In this method, we traverse each link $l$ downstream and place each of the first $n_{l}-1$ amplifiers as soon as it is possible to provide the maximum gain, subject to the constraints on the amplifier's output power. The remaining gain on the link is then obtained from the last amplifier. Hence, we need not let the power level of the signal die down to its lowest possible level. Thus, the per-wavelength input power to an amplifier may be greater than $p_{\text {sen }}$.
When the gain value $g \max _{l}$ is bounded by $G_{\max }$ (see Section II-C.1), there is a range of per-wavelength input power for which the amplifier can provide this gain. Hence, the position of the amplifier can be varied, while maintaining the desired gain.

2) ALAP (As Late As Possible): In this method, we traverse each link $l$ downstream and place each of the $n_{l}$ amplifiers only after the power level on each of the signals has fallen to its minimum acceptable value, viz. $p_{\text {sen }}$, unless, of course, we reach the end of the link. Again, each of the first $n_{l}-1$ amplifiers are operated at their maximum possible gain $\left(g \max _{l}\right)$.

For any link $l$ whose maximum amplifier gain, $g \max _{l}$ is bounded by $P_{\max }$, the placement is identical using both of the above methods. For the parameters in Table I and the amplifier gain model in Fig. 2, this holds for all links carrying more than 10 wavelengths, since from (5) we find that the maximum gain $\left(g \max _{l}\right)$ of an amplifier on link $l$ is bounded by the term containing $P_{\max }$ for $\left|\lambda_{l}\right|>10$. (This is true because $P_{\max }$ is 30 $\mathrm{dB}$ above (i.e., 1000 times greater than) $p_{\text {sen }}$, while the smallsignal gain $G_{\max }$ is $20 \mathrm{~dB}$ (i.e., it can provide a maximum amplification of 100 times to an input signal).)

For any link $l$, whose maximum amplifier gain $g_{m a x}$ is bounded by $G_{\max }$, we stand to benefit from ASAP because we can avoid placing amplifiers right next to each other as much as possible. This method helps in reducing the amplifiers' Amplified Stimulated Emission (ASE) noise which may affect the signals adversely. However, it is not clear if the choice of ASAP makes a significant difference in typical networks. We employ ALAP in Module IV and in our numerical examples for the sake of simplicity of coding, but this can be easily substituted with ASAP, if needed.

Several other methods of splitting the gain $\left(S G_{l}\right)$ along the link $l$, including uniform placement distribution among the $n_{l}$ amplifiers, are possible. Further discussions on various approaches to gain splitting can be found in [16]. A study on optimum amplifier location in spectrum-spliced WDM passive optical networks can be found in [17].

\section{F. Lower-Bound Analysis}

In this section, lower bounds on the number of amplifiers required for a given network to operate, using the link-by-link method (from [3]) and the global method (described in this paper), are derived. Note that, while these lower bounds may not always be achievable, they help characterize the nature of the two methods.

The link-by-link method in [3] forces the powers of all wavelengths to be equal to $p_{\text {sen }}$ at the beginning of all links originating at a star. The transmitter powers can be adjusted to avoid placing amplifiers on the links which originate at a station. We note that there are $L-N$ links in the network which originate at a star (recall that $L=$ number of links, $N=$ number of stations, and $M=$ number of stars); thus we obtain the lower bound of $L-N=2 \times(N+M-1)-N=$ $N+2 \times(M-1)$ on the number of amplifiers used by this method (Note that the number of unidirectional links in a "loopless" network with $N$ stations and $M$ stars is $2 \times$ (number of "nodes" -1$)=2 \times(N+M-1)$.). 


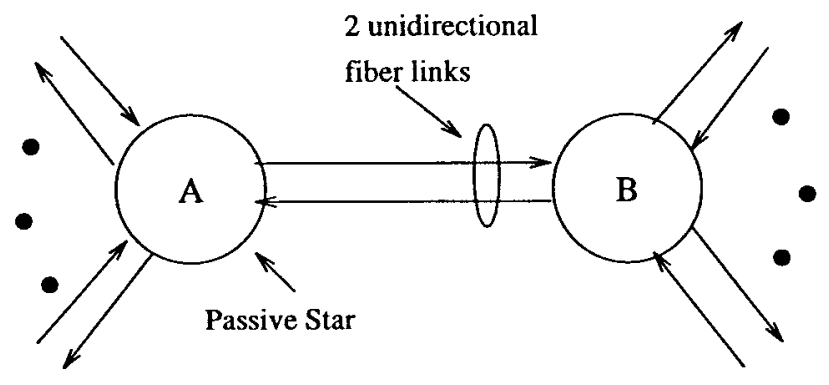

Fig. 8. A pair of adjacent stars in the network.

TABLE II

Comparison of the Amplifier Placement Algorithms

\begin{tabular}{l|c|c}
\hline Sample & \multicolumn{2}{|c}{ Number of Amplifiers } \\
\cline { 2 - 3 } & $\begin{array}{c}\text { Link-by-link } \\
\text { method [3] }\end{array}$ & $\begin{array}{c}\text { Global optimum } \\
\text { method (this paper) }\end{array}$ \\
\hline 1 (Fig. 1) & 79 & 77 \\
\hline 2 (Fig. 9) & 38 & 4 \\
\hline 3 (Fig. 10) & 113 & 18 \\
\hline
\end{tabular}

The global method discussed in this paper allows the powers on the wavelengths to be above $p_{\text {sen }}$. However, as shown below, this method requires that there is at least one amplifier between each pair of adjacent stars in the network. Consider one such pair of adjacent stars $(A$ and $B$ ) in the network (see Fig. 8), separated by a distance of $L_{A B} \mathrm{~km}\left(L_{A B}>0\right)$. Let $p_{A}$ and $p_{B}$ be the per-wavelength powers of the signals at the output of the stars $A$ and $B$, respectively. Let $D_{A}$ and $D_{B}$ be the corresponding degrees of the stars $A$ and $B$. Let us examine if it is feasible to have no amplifiers on either link between the stars $A$ and $B$. Consider the link from star $A$ to star $B$. The signal on any particular wavelength exits star $A$ at a power $p_{A}$ and then suffers attenuation along the link from $A$ to $B$ and a splitting loss at star $B$, and finally exits star $B$ at a power $p_{B}$. Thus, we have

$$
p_{A}-\alpha \cdot L_{A B}-10 \log _{10}\left(D_{B}-1\right)=p_{B}
$$

i.e.,

$$
p_{A}>p_{B}
$$

Similarly, considering the link from star $B$ to star $A$, we have

$$
p_{B}-\alpha \cdot L_{A B}-10 \log _{10}\left(D_{A}-1\right)=p_{A}
$$

i.e.,

$$
p_{B}>p_{A} .
$$

Equations (18) and (19), contradict each other! Hence, our assumption that there are no amplifiers required on the links between $A$ and $B$ is false. Thus, there must be at least one amplifier on either of these links. Similarly, there should be at least one amplifier between all other pairs of adjacent stars in the network. Since the $M$ stars in the network form a "tree" (which is a "loopless" network), the number of pairs of adjacent stars in the tree network is $M-1$. Thus, the lower bound on the number of amplifiers required by this method is $M-1$, where $M$ is the number of stars in the network.

\section{NUMERICAL EXAMPLES}

\section{A. An Illustrative Example: Sample Network 1}

Consider the Sample Network 1 in Fig. 1. This is similar to the example network studied in [3] except that the size of group 3 has been decreased from 35 to 28 to make the network feasible for the values of $P_{\max }=0 \mathrm{dBm}$ and $p_{\mathrm{sen}}=-30$ $\mathrm{dBm}$. In this regard, note that the corresponding original example network in [3] was infeasible for its choice of network parameters.

The network has $N=63$ stations and $M=4$ passive stars. The number of amplifiers required using the algorithm in [3] is 79 . The optimum number of amplifiers as obtained by our algorithms in Section II is 77. The difference in the number of amplifiers is not significant for this topology. This is due to the fact that the access stations are so far away $(20 \mathrm{~km})$ from the stars that they prevent our optimization algorithms from eliminating the amplifiers on the access links. However, if we reduce the distance of the stations from the stars to $7.13 \mathrm{~km}$, our global optimum method uses only 62 amplifiers against the 79 used by [3], a $21 \%$ savings! This savings of these 15 amplifiers (from 77 to 62) occurs at the access links from star 3 to the stations in group 2. The number of amplifiers reduces to 42 (a further savings of 20 amplifiers!) if the distance between the stations and their corresponding stars is further reduced to $3.27 \mathrm{~km}$; at this distance, the amplifiers on the access links from star 1 to the stations in group 1 are eliminated. The Sample Network 1 is chosen only for comparison purposes. Two other sample ("typical") networks that we will also examine are shown in Figs. 9 and 10.

We describe below the operation of the four modules with the Sample Network 1 as the input network topology. Module I tests the feasibility of the network given the device parameters (see Table I). Module II generates the constraints on the power levels throughout the network. Module III uses a MILP solver to minimize the number of amplifiers in the network. Module IV provides the actual placement of the amplifiers on the various links in the network.

Module I tests the feasibility of the network by checking whether the inequality (2) holds good for this network. As mentioned in Section II-B, we need only consider the star $i_{\max }$ and its input link $j_{\max }$ for which the product $\left(D_{i_{\max }}-\right.$ 1) $\left(\left|\lambda_{j_{\max }}\right|\right)$ is the maximum among all such pairs in the network. Consider the link 5 from star 2 to star 4 in Fig. 1. This link carries 35 wavelengths $\left(\left|\lambda_{5}\right|\right)$ into star 4 from groups 1 and 2 . Also, star 4 has a degree $\left(D_{4}\right)$ of 29 , and the product, $\left(D_{4}-1\right)\left(\left|\lambda_{5}\right|\right)=980$, is the maximum among such pairs in the entire network. Hence, for this network, $i_{\max }=4$ and $j_{\max }=5$. This network is feasible since (2) holds for $i=i_{\max }$ and $j=j_{\max }$.

Module II generates the constraints from the input for this particular network. The complete MILP formulation for this network can be found in [18].

Module III contains the MILP solver and outputs the optimum value of the objective function and the variables $\left(p_{i}\right.$ 's, $n_{l}$ 's and $S G_{l}$ 's).

The amplifier placement using the algorithm in [3] is shown in Fig. 11. The optimum amplifier placement generated by 


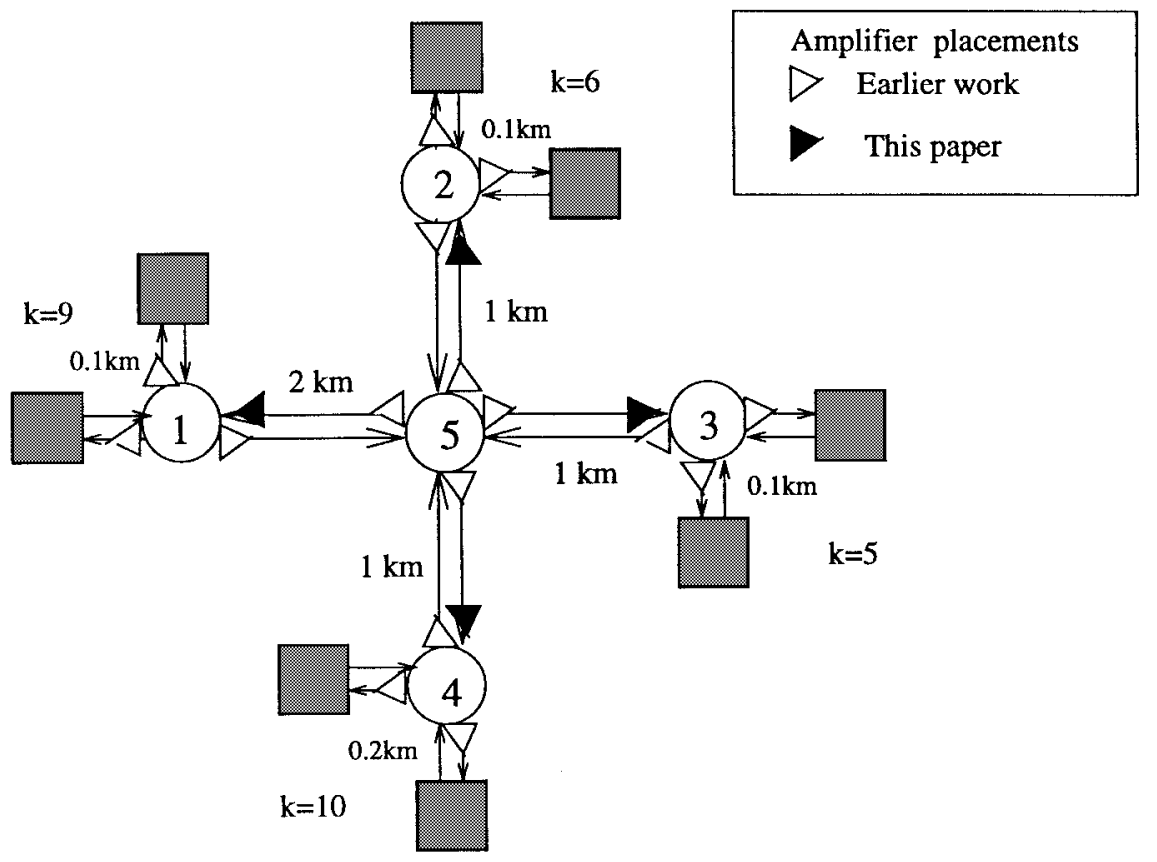

Fig. 9. Sample Network 2.

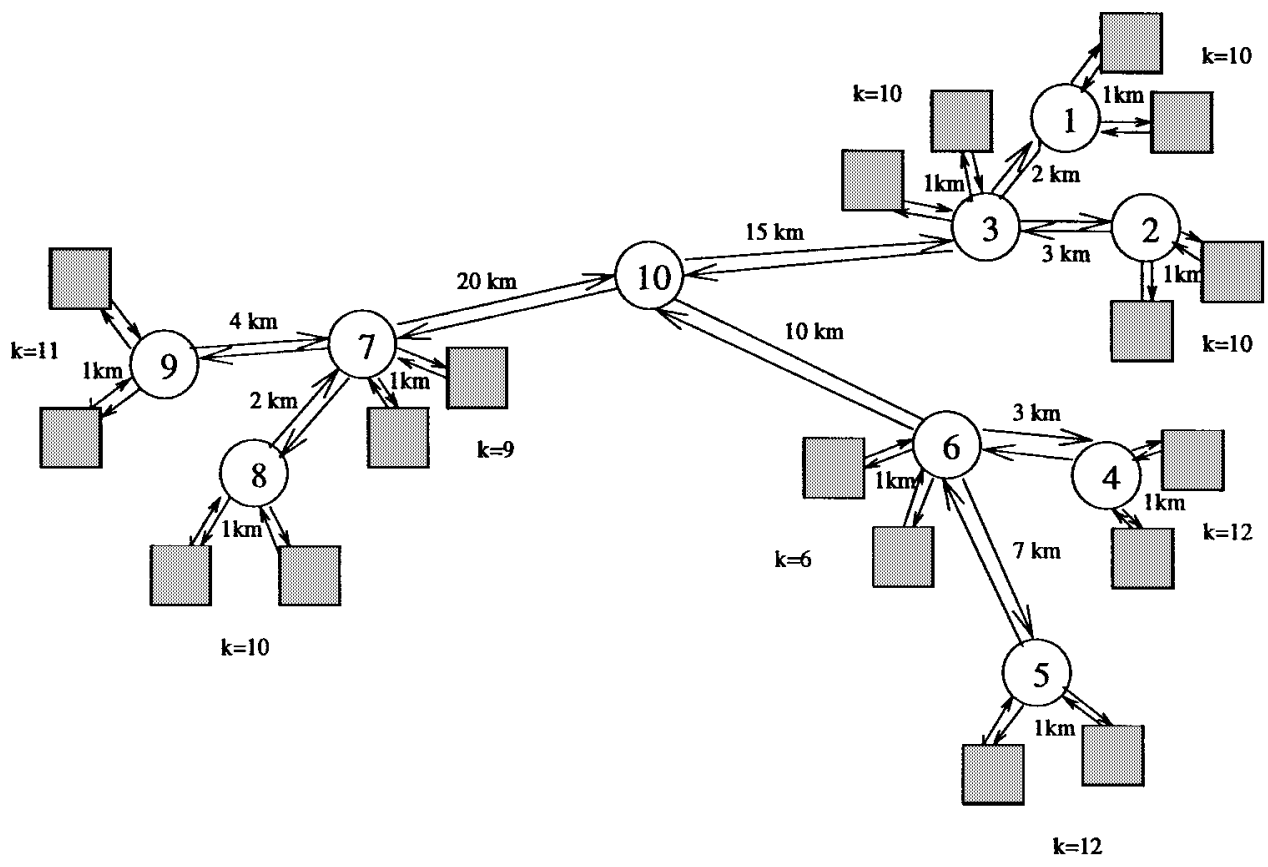

Fig. 10. Sample Network 3.

Module IV is shown in Fig. 12. Other details such as precise amplifier locations have been omitted for clarity in the figures and can be found in [18].

\section{B. Results and Discussion}

We compare the number of amplifiers used by our global optimum algorithm versus the number of amplifiers used by the link-by-link approach in [3] for three sample networks. The networks are shown in Figs. 1, 9, and 10. Network 1 represents a feasible network adapted from the example used in [3]. Network 2 represents a broadcast network spread over a small geographical area (e.g., within a college campus). Network 3 represents a network spread over a larger geographical area (e.g., within a city or a metropolis). In both Sample Networks 2 and 3, $k$ represents the size of a group of access stations.

The results are presented in Table II. In Fig. 9, the empty triangles represent the amplifiers placed using the algorithm in [3] whereas the filled triangles represent the amplifiers placed using our optimum method.

We notice that our global optimum method performs much better than the link-by-link method in [3], especially for networks with short access links. We explain the difference below. The link-by-link method was designed to equalize the power levels of the wavelengths in the network, as opposed to 


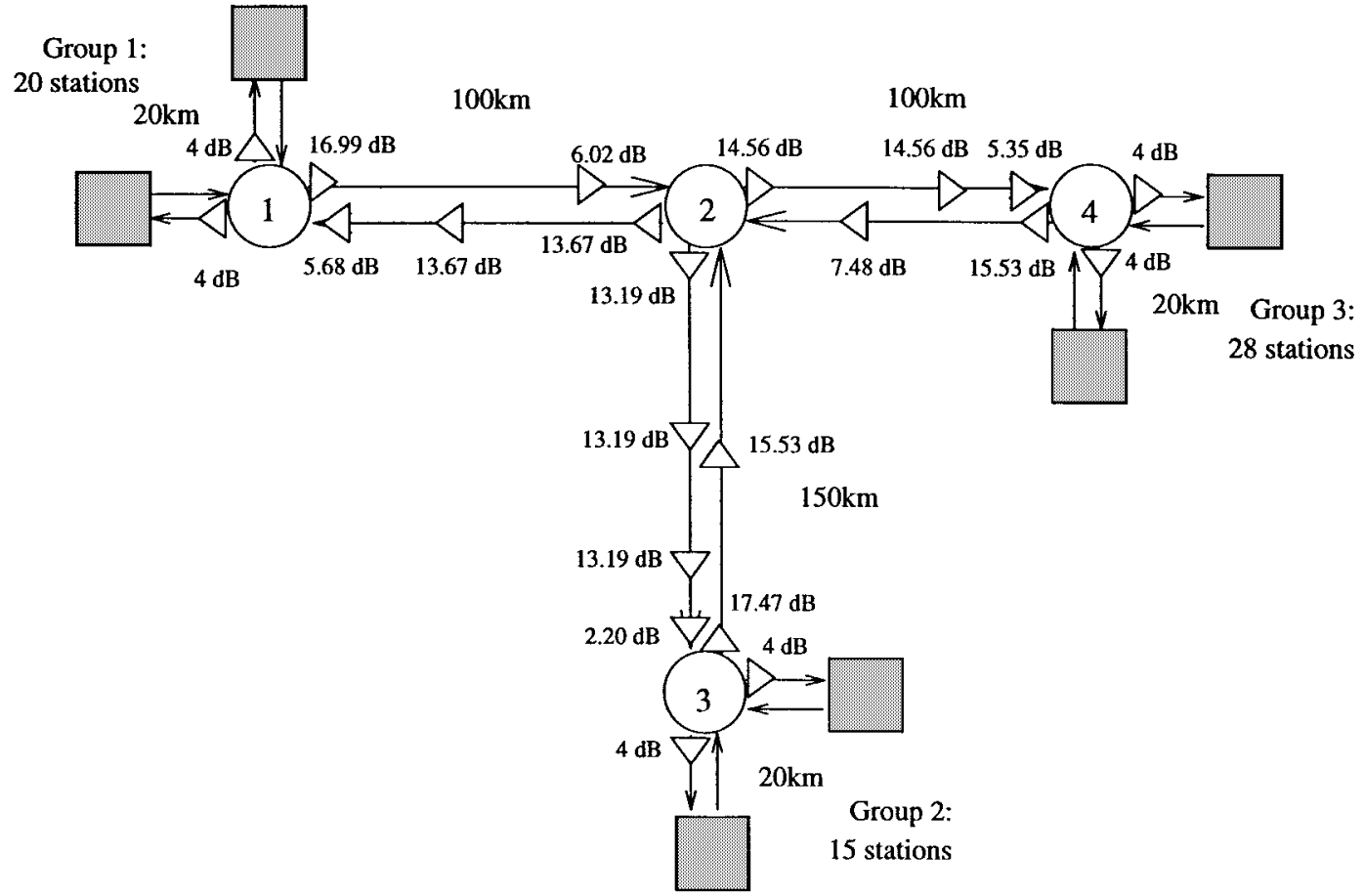

Fig. 11. Amplifier placments for Sample Network 1 using the link-by-link method [3].

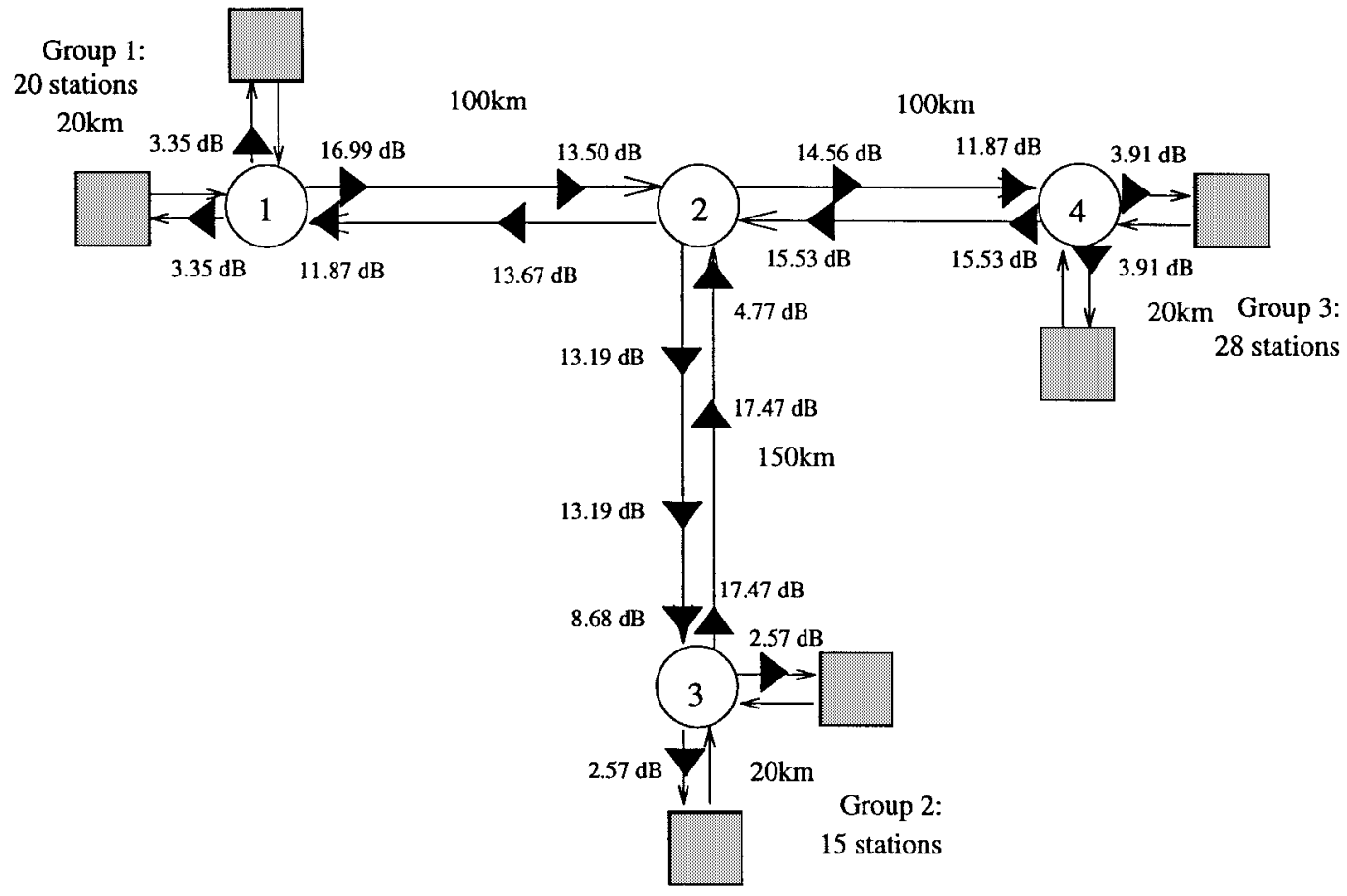

Fig. 12. Amplifier placements for Sample Network 1 using the global optimum method (this paper).

trying to minimize the number of amplifiers in the network. By forcing the power levels of all wavelengths to be equal to $p_{\text {sen }}$ at the beginning of most links (all links except those from stations to stars), the link-by-link method fixed the power levels at the end of each link too. This led to a very simple amplifier-placement algorithm which operated on each link individually (locally) without regard to the signal powers on other links.
The global optimum method introduced in this paper, however, allows wavelengths at the beginning of the links to be above the absolute minimum power level allowed, namely, $p_{\text {sen }}$. In fact, the algorithm computes the minimum power level needed by the wavelengths at the beginning of each link in order to minimize the total number of amplifiers used in the network. The savings (in number of amplifiers and hence, the network cost) is greater for networks that have short access 
links because the global optimum method can usually avoid placing an amplifier on a short link simply by exiting the preceding star with enough power to traverse the short link. As we observe from Fig. 9, the global optimum method "pushes" the amplifiers toward the upstream side of the stars (away from the access links), thereby compensating for the splitting loss in advance; a single amplifier on the upstream side eliminates the need for several amplifiers on each of the access links. From Figs. 11 and 12, we notice that even in the case of networks with long access links (which require amplification on each one of them), the global optimum method is able to save a few amplifiers on the internal links between stars.

\section{CONCLUSION}

We considered the problem of achieving power equalization in an optical network using the minimum number of optical amplifiers. First, a network feasibility check was conducted to determine if the network could be made operational. Then, a set of constraints for the problem was generated, and the corresponding mixed-integer linear program (MILP) was solved using the package lp_solve. Finally, an amplifierplacement algorithm was run to determine the exact placement of the optical amplifiers. Numerical examples showed that this scheme has the potential to save a significant number of amplifiers over the scheme in [3], which just attempted to perform power equalization. With optical amplifiers remaining very expensive, our scheme can significantly reduce network costs.

We consider elsewhere [10] the problem of minimizing the number of amplifiers without the constraint on power equalization. This approach may result in utilizing fewer amplifiers under certain circumstances. However, retaining this constraint results in a simpler formulation and an exact solution as shown in this paper. In the future, we plan to employ improved models of the optical amplifier [including the popular erbium-doped fiber amplifier (EDFA)], incorporating the wavelength-dependent gain, gain saturation, and noise characteristics described in [13]. We also plan to expand our current methods to handle switched networks and the unique problems that arise in this new environment.

\section{REFERENCES}

[1] J. R. Stern, J. W. Ballance, D. W. Faulkner, S. Hormung, D. B. Payne, and K. Oakley, "Passive optical networks for telephony applications and beyond," Electron. Lett., vol. 23, pp. 1255-1257, 1989.

[2] N. J. Frigo, P. P. Iannone, P. D. Magill, T. E. Darcie, M. M. Downs, B. N. Desai, U. Koren, T. L. Koch, C. Dragone, H. M. Presby, and G. E. Bodeep, "A wavelength division multiplexed passive optical network with cost-shared components," IEEE Photon. Technol. Lett., vol. 6, pp. $1265-1367,1994$.

[3] C.-S. Li, F. F.-K. Tong, C. J. Georgiou, and M. Chen, "Gain equalization in metropolitan and wide area optical networks using optical amplifiers," in Proceedings, IEEE INFOCOM '94, Toronto, Ont., Canada, June 1994, pp. $130-137$.

[4] P. P. Iannone, N. J. Frigo, and T. E. Darcie, "A WDM PON architecture with bidirectional optical spectral splicing," in Tech. Dig., Optic. Fiber Commun. Conf. (OFC 95), San Diego, CA, vol. 8, pp. 51-53, Feb. 1995.

[5] J. M. Senior, A. J. Phillips, M. S. Leeson, and R. Johnson et al., "Upgrading superPON: Next step for future broadband access networks," in Proc. SPIE-The Int. Soc. Optic. Eng., Boston, MA, vol. 2919, pp. 260-266, Nov. 1996.

[6] V. Tandon, M. Wilby, and F. Burton, "A novel upgrade path for transparent optical networks based on wavelength reuse," in Proc., IEEE
INFOCOM '95, Apr. 1995, pp. 1308-1315.

[7] S. B. Alexander et al., "A precompetitive consortium on wide-band all-optical networks," J. Lightwave Technol., vol. 11, pp. 714-735, May/June 1993.

[8] K. Magari, M. Okamoto, and Y. Noguchi, "1.55 $\mu \mathrm{m}$ polarization insensitive high gain tensile strained barrier MQW optical amplifier," IEEE Photon. Technol. Lett., vol. 3, pp. 998-1000, Nov. 1991.

[9] M. Soulliere, "Standards activities for WD line systems and optical networks," in Proc. Third IEEE COMSOC Workshop WDM Network Management and Control-ICC '97, Montreal, P.Q.. Canada, June 1997.

[10] B. Ramamurthy, J. Iness, and B. Mukherjee, "Minimizing the number of optical amplifiers needed to support a multi-wavelength optical LAN/MAN," in Proc. IEEE INFOCOM '97, Kobe, Japan, Apr. 1997.

[11] A. E. Siegman, Lasers. New York: University Science Books, 1986.

[12] K. Inoue, "Crosstalk and its power penalty in multichannel transmission due to gain saturation in a semiconductor laser amplifier," J. Lightwave Technol., vol. 7, pp. 1118-1124, July 1989.

[13] C. R. Giles and E. Desurvire, "Modeling erbium-doped fiber amplifiers," J. Lightwave Technol., vol. 9, pp. 271-283, Feb. 1991.

[14] M. Berkelaar, lp_solve: A Mixed Integer Linear Program Solver, available from ftp://ftp.es.ele.tue.nl/pub/lp_solve/.

[15] G. V. Reklaitis, A. Ravindran, and K. M. Ragsdell, Engineering Optimization: Methods and Applications. New York: Wiley, 1983.

[16] H.-D. Lin, "Gain splitting and placement of distributed amplifiers," Tech Rep. RC 16216 (\#72010), IBM, Oct. 1990.

[17] M. J. L. Cahill, G. J. Pendock, M. A. Summerfield, A. J. Lowery, and D. D. Sampson, "Optimum optical amplifier location in spectrum-sliced WDM passive optical networks for customer access," in Optic. Fiber Commun. (OFC '98) Tech. Dig., San Jose, CA, Feb. 1998, vol. 2, pp. 403-404.

[18] B. Ramamurthy, "Efficient design of wavelength division multiplexing (WDM)-based optical networks," Ph.D. dissertation, Dep. Comput. Sci., Univ. California, Davis, CA, July 1998.

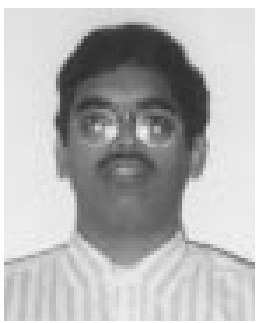

Byrav Ramamurthy received the B.Tech. degree in computer science and engineering from the Indian Institute of Technology, Madras, India, in 1993. He received the M.S. and Ph.D. degrees in computer science from the University of California (UC), Davis, in 1995 and 1998, respectively.

Since August 1998, he has been Assistant Professor in the Department of Computer Science and Engineering at the University of Nebraska-Lincoln. His research interests include high-speed networks, distributed systems, and telecommunications. $\mathrm{He}$ was a recipient of the Indian National Talent Search Scholarship and was a Fellow of the Professors for the Future Program at UC Davis.

Jason Iness received the Ph.D. degree in computer science from University of California (UC), Davis, in 1997.

Since 1997, he has been with the Intel Corporation, Portland, OR.

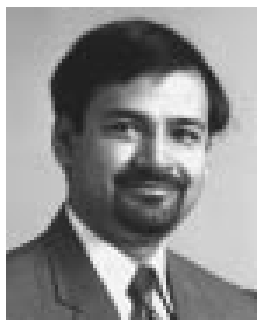

Biswanath Mukherjee (S'82-M'87) received the B.Tech. (Honors) degree from the Indian Institute of Technology, Kharagpur, India, in 1980 and the $\mathrm{Ph} . \mathrm{D}$. degree from the University of Washington, Seattle, in June 1987.

At the University of Washington, he held a GTE Teaching Fellowship and a General Electric Foundation Fellowship. In July 1987, he joined the University of California (UC), Davis, where he has been a Professor of Computer Science since July 1995, and Chairman of Computer Science since September 1997. His research interests include lightwave networks, network security, and wireless networks.

$\mathrm{He}$ is co-winner of paper awards presented at the 1991 and 1994 National Computer Security Conferences. He serves on the Editorial Boards of the IEEE/ACM TRANSACtions on NeTWorking, IEEE Network, ACM/Baltzer Wireless Information Networks (WINET), Journal of High-Speed Networks, and Photonic Network Communications. He served as the Technical Program Chair of the IEEE INFOCOM'96 Conference. He is the author of the textbook Optical Communication Networks (New York: McGraw Hill, 1997), a book which received the Association of American Publishers, Inc.'s, 1997 Honorable Mention in Computer Science. 